\title{
Il futuro della Sanità: sostenibilità finanziaria e scenari evolutivi
}

\author{
The future of Healthcare: financial sustainability and possible scenarios
}

\section{Piero Cappelletti}

Ricevuto: 15 giugno 2012 / Accettato: 15 giugno 2012

(C) Springer 2012

Riassunto Uno sguardo all'evoluzione dei sistemi organizzativi della Sanità è necessario per comprendere le prospettive, gli scenari e i ruoli della Medicina di Laboratorio nell'ambito dell'odierna crisi economica e culturale e nel prossimo futuro. Negli ultimi anni, l'imperativo di ridurre i costi migliorando gli esiti di salute, presente fin dalle crisi inflazionistiche degli anni ' 70 e ' 80 del secolo scorso, si è fatto più stringente e condiziona l'evoluzione della Sanità.

In campo europeo, studi sugli scenari possibili definiscono traiettorie che vanno dalla completa o parziale privatizzazione dei sistemi a soluzioni basate sulla priorità della prevenzione o dei centri d'eccellenza pan-europei oppure a scenari determinati dal trionfo della tecnologia e della concezione della salute come motore economico.

A livello italiano lo studio più approfondito punta sulle aree di possibile discontinuità ambientale: la disponibilità complessiva e le fonti della disponibilità finanziaria; i confini del welfare e la mission delle aziende; la cul-

Piero Cappelletti (ه)

IRCCS CRO Aviano

Via Franco Gallini 2, 33081 Aviano (PN)

piero.cappelletti@cro.it tura, i profili di consumo, la propensione alla mobilità dei pazienti; la trasformazione del capitale umano e dei modelli organizzativi delle aziende; la rivoluzione tecnologica e le nuove architetture infrastrutturali; la strutturazione (ospedali versus territorio) del Servizio Sanitario Nazionale (SSN). Il focus principale è sull'evoluzione delle aziende sanitarie.

Esistono in Europa tentativi di intervento che non sono il mero razionamento: la riorganizzazione strutturale dei sistemi sanitari in termini di concentrazione/dispersione delle tecnologie e interventi sulla base della complessità; il contributo dei pazienti alla prevenzione e agli stili di vita per una crescita culturale; l'intelligente uso della medicina "remota" sulla base di una moderna ITC.

Un tema emergente è quello del personale sanitario, chiamato a grandi cambiamenti perché cambiano le tecnologie e le potenzialità, cambiano i pazienti e le loro richieste, cambia la loro vita lavorativa in quanto si allunga e in quanto le competenze professionali mutano continuamente, richiedendo una crescita professionale continua e la capacità di travalicare i tradizionali confini delle mansioni originarie.

Parole chiave Sistemi sanitari $\cdot$ Sostenibilità economica - Riorganizzazione

Summary To understand the present and the future of Laboratory Medicine, we must understand the evolution of the healthcare. In present years the healthcare imper- 
ative - "lowering costs and improving outcomes" becomes more and more stringent.

In Europe the trends of development of healthcare were described as contrasting but not mutually exclusive scenarios: total or partial privatization of healthcare systems, "wellness first" model, pan-European system of excellence centers, "technology triumphant" scenario.

In Italy the best survey about future of healthcare pointed out the institutional and financial issues, the interrelations regarding mobility of patient, models of care and health organization and the relationship connecting technology, logistic and organization. The focus is on development of healthcare trusts.

In Europe some initiatives exist for a re-organization of healthcare and not only for rationing of care. The examples are mutually re-enforcing and are based on reshaping organization framework, centrality of ITC, empowerment of patients, and change of minds and competences of workforce.

Key words Healthcare . Financial sustainability . Reorganization

\section{Introduzione}

La trasformazione della Medicina di Laboratorio nel ventunesimo secolo non è facilmente comprensibile, come ricordano Parker e Talbert [1], se non la si colloca all'interno dell'evoluzione culturale del Mondo e dello sviluppo scientifico e tecnologico della Medicina e della sua organizzazione sociale (Fig. 1).

Dal punto di vista dell'evoluzione culturale, gli ultimi decenni sono stati caraterizzati dall'irrompere del "soggetto" nell' arena filosofica e sociale, che è stato un fattore principale nel determinare l'attuale crisi della Medicina [2]. La risposta dell'organizzazione sanitaria è stata quella di porre al centro dell'organizzazione assistenziale il paziente-cittadino [3] (o perlomeno di dichiarare tale volontà), stimolata anche dalla consapevolezza

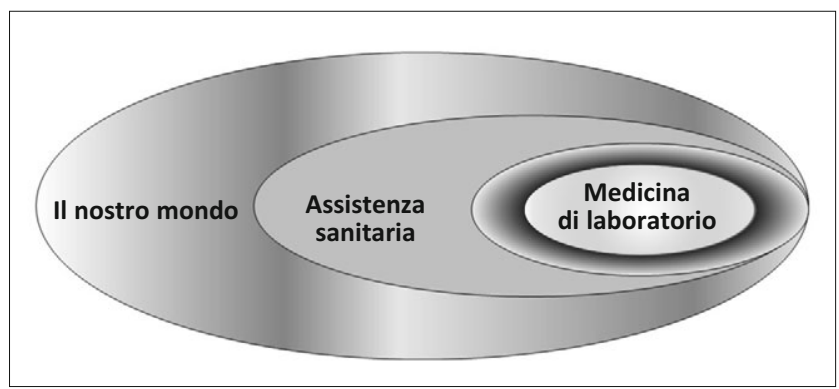

Fig. 1 Nel ventunesimo secolo la Medicina di Laboratorio continua a essere influenzata dai cambiamenti dell'organizzazione sanitaria e dall'andamento del mondo. Mod. da Parker e Talbert [1] degli specifici diritti che gli competono [4]. Tuttavia, tale processo si è scontrato, da un lato, con le resistenze derivanti dall'entropia dell' organizzazione sanitaria e, dall'altro, con le ricorrenti carenze finanziarie. L'imperativo sanitario di migliorare gli esiti di salute, riducendo i costi, è divenuto via via sempre più stringente [5].

In questo Editoriale si cercherà di delineare sinteticamente quali sono gli elementi culturali ed economici che possono influenzare il futuro delle organizzazioni sanitarie di cui la Medicina di Laboratorio fa parte. Niels Böhr diceva ironicamente che fare previsioni è assai difficile, in particolare per quel che concerne il futuro, e ciò pare perfettamente calzante per le organizzazioni sanitarie. Si conoscono sufficientemente bene i fattori critici dell'evoluzione dei sistemi sanitari: l'invecchiamento della popolazione e le sue conseguenze sulla patologia, lo sviluppo di nuove tecnologie, l'aumento della complessità delle organizzazioni sanitarie, l'affermazione di nuove professionalità e le modifiche quantitative e qualitative dei gruppi professionali, la crescente limitatezza delle risorse finanziarie. Tuttavia, si comprendono con difficoltà le interrelazioni e il peso relativo dei fattori critici. Di conseguenza, si possono intraprendere due diverse strategie: si utilizzano solo gli elementi meno incerti (se ne vedrà un esempio per quanto attiene l'aspetto finanziario) oppure si utilizza il metodo degli "scenari", che "non sono previsioni del futuro, quanto piuttosto anticipazioni che intendono esplorare il futuro per generare visioni, facilitare interpretazioni di fenomeni in evoluzione, porre dubbi da sciogliere" per i prossimi 10-30 anni [6].

\section{La sostenibilità finanziaria}

Il tema della progressiva carenza di risorse finanziarie per la Sanità deriva dal continuo incremento esponenziale della richiesta di prestazioni e, più volte negli ultimi quarant'anni, dalla contrazione delle disponibilità derivanti dalle crisi ricorrenti dello sviluppo economico capitalista. L'analisi delle prospettive di finanziamento della Sanità, dato per scontato che debba contrarsi, sono rese incerte dalla difficoltà di misurare perfettamente la spesa attuale e, a maggior ragione, i fabbisogni futuri legati a una molteplicità di fattori di difficile determinazione.

Per quanto riguarda l'Italia, se si utilizzano i dati ufficiali di spesa [7], si può affermare che nel 2010 la spesa sanitaria totale (pubblica e privata) è stata di 148,2 miliardi di euro, pari al 9,6\% del Prodotto Interno Lordo (PIL). Nel 2009 la spesa sanitaria totale è stata pari al 9,5\% del PIL. Nel 2010 la spesa complessiva effettiva del SSN (Servizio Sanitario Nazionale), cioè la spesa pubblica, è stata di 111,168 miliardi di euro, con un aumento dello $0,9 \%$ rispetto ai 110,219 miliardi di euro del 2009 . 
Il rapporto spesa complessiva SSN/PIL si attesta al 7,2\% nel 2010. Nel 2009 tale rapporto è stato pari al 7,3\%. Nel 2010 il finanziamento del SSN è stato pari a 108,842 miliardi di euro, cresciuto dell'1,8\% rispetto ai 106,967 miliardi di euro del 2009. Il disavanzo sanitario nel 2010 è stato di 2,3 miliardi di euro. Nel 2009, il disavanzo maggiore, pari all' $89 \%$ del totale, appare collocato nelle regioni del centro e del sud (Lazio, Campania, Puglia, Sicilia, Calabria e Sardegna). Con 1374 milioni di euro il Lazio detiene il 39,8\% del disavanzo totale 2009.

Con questi dati, l'Italia si colloca in perfetta media con i Paesi OECD (Organisation for Economic Co-operation and Development) [8] per percentuale di PIL speso in Sanità $(9,5 \%)$, ma nell'utilizzo di questo indicatore si deve tenere conto che l'impennata degli anni recenti (2007: $8,7 \% ; 2008: 9,0 \% ; 2009: 9,5 \% ; 2010: 9,6 \%)$ è ampiamente dovuta alla recessione in atto e al conseguente rallentamento/diminuzione dell'andamento del PIL. Un indicatore migliore è la spesa per abitante, che anch'essa si colloca in media OECD (3137 USD Italia versus 3223 USD OECD nel 2009), pari a circa 3000 euro, comprensivi dei costi sanitari e sociali [9], e la cui struttura è presentata in Figura 2. A carico dell'INPS devono essere assegnati altri 371 euro per residente destinati a integrazione del reddito, per una stima totale complessiva di $€ 3373$ pro capite. Utilizzando questo indicatore, la crescita della spesa pro capite in Italia è cresciuta in termini reali dell'1,6\% per anno tra il $2000 \mathrm{e}$ il 2009, ben sotto la crescita OECD (mediamente del $4,0 \%$ per anno nello stesso periodo).

La quota pubblica è, come nella maggior parte dei Paesi dell'OECD, del tutto maggioritaria, ma in Italia rappresenta circa l' $80 \%$ della spesa contro il $71,7 \%$ della media OECD, percentuale inferiore solo ai Paesi scandinavi, al Regno Unito e al Giappone.

Sulla spesa cosiddetta sanitaria pesa per una quota importante il costo sociale $(23,5 \%)$. La spesa sanitaria in

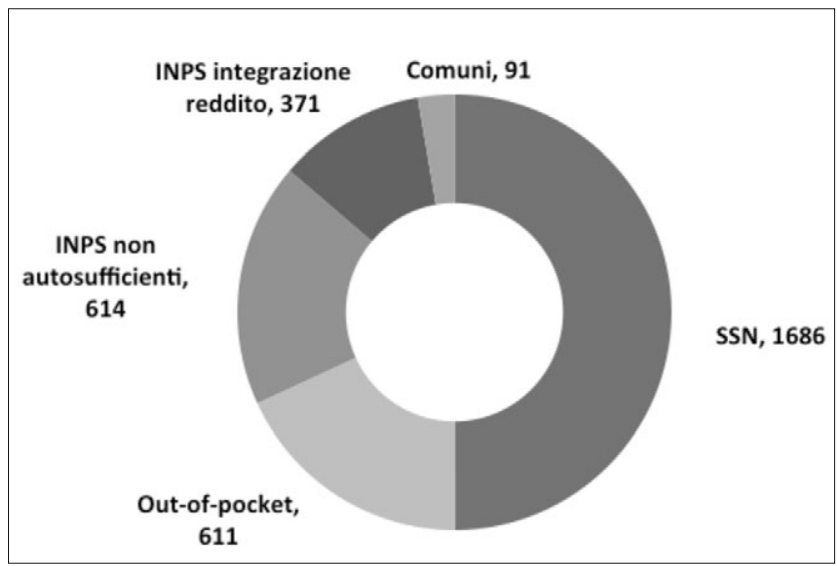

Fig. 2 Spesa annua pro capite in euro $(3373 €)$ in Italia nel 2010. Mod. da Longo e Tasselli [9] senso stretto (2297 euro pro capite nel 2010) è sostenuta per il $73,5 \%$ dallo Stato e dalle Regioni e per il $26,5 \%$ dai cittadini. Negli ultimi tre anni il tasso di incremento della spesa privata è stato superiore di quello per la spesa pubblica (2,9\% versus $2,3 \%$ per anno composto).

La spesa sanitaria pubblica (SSN) rappresenta il 7,2\% del PIL nel 2010, secondo la Corte dei Conti, e il 7,3\% secondo i dati Istat revisionati da The European House Ambrosetti [10] e comprendenti l'assistenza protesica e balneotermale, "altra" assistenza e servizi amministrativi per 113.457 milioni di euro (Fig. 3). Nel 2011 la spesa sanitaria pubblica ha rappresentato il $6,9 \%$ secondo il CeRM (Competitività, Regolazione, Mercati, un gruppo indipendente di ricerca, nato nel 2003 per stimolare riforme strutturali per la crescita) [11] e il 7,3\% secondo il Ministero dell'Economia e delle Finanze (MEF) [12]. Come si evince da tali dati, i valori assoluti e percentuali della spesa pubblica variano secondo le fonti, in ragione sia delle voci contemplate sia dei criteri di aggregazione, quali i SHA (Systems of Health Accounts) dell'OCSE, quelli europei EPC-WGA (Economic Policy Committee Working Group on Ageing), o quelli nazionali.

Le previsioni di andamento della spesa sanitaria, per la complessità del fenomeno, ignorano le cosiddette "componenti esogene" (le scoperte scientifiche e lo sviluppo tecnologico, il loro impatto sull'efficacia e sui

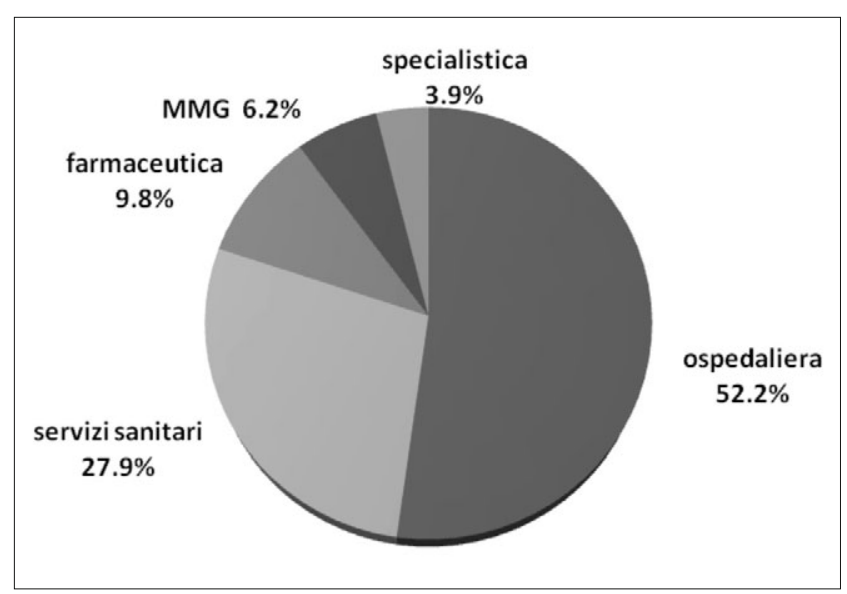

Fig. 3 Struttura della spesa sanitaria pubblica in Italia nel 2010, secondo The European House-Ambrosetti. Mod. da [10]

costi delle prestazioni sanitarie, le politiche di contenimento della spesa e le scelte in tema di organizzazione dei servizi e di investimento), per la loro non prevedibilità, la discrezionalità degli interventi e l'elevato grado di discontinuità indotta, che rende assai difficile quantificarne gli effetti. Pertanto, i fattori di cui si tiene conto sono quelli demografici (variazione della numerosità della popolazione e suo mix per sesso e fasce d'età) e quelli macroeconomici (incremento della spesa sanitaria come conseguenza dell' aumento di disponibilità di reddi- 
to, elasticità della spesa sanitaria pro capite rispetto alla variazione di reddito disponibile). Gli studi macroeconomici mostrano che il driver principale è la variazione di mix demografico (l'aumento delle fasce d'età più avanzate), in cui si concentra la maggiore necessità di spesa per il morbidity burden connesso (negli ultra 65 anni, che rappresentano oggi circa il $22 \%$ della popolazione, è concentrato il $50 \%$ della spesa; l'ultimo anno di vita costa fino a 20 volte di più dei precedenti per costi sanitari). D'altra parte, nelle società più avanzate vi è una propensione a una spesa sanitaria pro capite superiore alla crescita del PIL (elasticità $>1$ ).

Le metodologie adottate per la previsione possono variare. La previsione del rapporto fra spesa sanitaria pubblica e PIL effettuata nel 2011 dal MEF [12] si basa sulle indicazioni metodologiche concordate in ambito EPC-WGA. Si assume che la struttura dei consumi specifici per età, sesso e tipologia di prestazione resti costante per l'intero periodo di previsione, mentre la dinamica del Consumo Pro capite Standardizzato (CPS), che esprime la variazione del consumo sanitario a parità di struttura demografica, evolva in linea con il PIL pro capite. Tale approccio metodologico è denominato "pure ageing scenario", in quanto rende le variazioni del rapporto spesa sanitaria/PIL dipendenti esclusivamente dalla modificazione della struttura della popolazione.

Tuttavia, in considerazione dell'esistenza di fattori esplicativi diversi da quelli riconducibili alla sola dimensione demografica, sono state analizzate differenti ipotesi di evoluzione del consumo sanitario che riflettono il miglioramento delle condizioni di salute correlato con l'aumento della speranza di vita, i differenziali inflazionistici fra prodotti sanitari e prodotti non sanitari, anche in dipendenza del diverso contenuto tecnologico e l'effetto trainante dei costi sanitari esercitato dalla remunerazione del fattore lavoro. La combinazione delle ipotesi alternative, che prende il nome di "reference scenario", prevede la dinamicità del profilo per età del consumo sanitario, realizzata tramite la metodologia del "death related costs" (poiché è nell'ultimo anno di vita che si concentra la più alta percentuale di spesa sanitaria; gli anni di vita guadagnati pesano molto relativamente) per la componente ospedaliera e del "dynamic equilibrium" (che assume che gli anni di vita guadagnati siano di buona salute, applicato nella misura del $50 \%$ della variazione della speranza di vita) per le altre prestazioni sanitarie, l'elasticità del CPS al PIL pro capite superiore all'unità, per la componente acute della spesa sanitaria e l'aggancio del CPS alla produttività, per la componente LTC (Long Term Care) (spesa per anziani e non autosufficienti) della spesa sanitaria, che qui non verrà trattata.

Nell'ipotesi del "pure ageing scenario", la previsione del rapporto fra spesa sanitaria pubblica e PIL mostra una crescita piuttosto regolare fra il 2010 e il 2040. Nell'ultima parte del periodo di previsione, il ritmo di crescita presenta una leggera flessione dovuta all'uscita delle generazioni del baby boom. Complessivamente, il rapporto si incrementa di circa 1,5 punti percentuali, passando dal 7,3\% del 2010 all' $8,8 \%$ del 2060 . La previsione coerente con le ipotesi del "reference scenario" presenta andamenti sostanzialmente sovrapponibili in conseguenza della quasi totale compensazione tra l'effetto di contenimento indotto dalle ipotesi sui profili dinamici del consumo sanitario e quello espansivo prodotto dalle ipotesi sul CPS. Solo nella parte finale del periodo di previsione il primo effetto tende a prevalere leggermente sul secondo. Le previsioni sulla base dei criteri nazionali ed EPC-WGA sono sovrapponibili). L'applicazione di scenari alternativi al "pure ageing" contiene l'incremento all'8,3\% nel 2060.

Secondo The European House - Ambrosetti [10], la proiezione della spesa sanitaria al 2050, sulla base dei fattori età e PIL, conduce a una spesa di 281,5 miliardi di euro, pari al 9,7\% del PIL. Secondo il CeRM [11], in uno scenario caratterizzato da mix demografico e contenuta elasticità, la spesa standard passa dal 7,3\% del PIL nel 2015 a poco meno dell' $8,0 \%$ nel 2030 , quella lorda da poco meno del $7,6 \%$ all' $8,3 \%$; nello scenario caratterizzato da elevata elasticità, seppure corretto sul costo degli anni di vita guadagnati, la spesa standard passa dal $7,3 \%$ del PIL nel 2015 a poco più dell' $8,4 \%$ nel 2030 , quella lorda dal $7,6 \%$ a oltre 1 ' $8,7 \%$. All'interno di questa media, lo spaccato regionale appare molto eterogeneo. Nel 2030, nel Mezzogiorno l'incidenza della spesa standard sarà almeno pari al $10 \%$, con punte che supereranno l'11\%.

È del tutto evidente che, al di là degli orizzonti temporali $(2030,2040,2050,2060)$ e delle metodologie adottate, la crescita della spesa sanitaria negli anni futuri sarà insostenibile $(+2,7 \%$ per anno fino al 2050 per la quota di spesa pubblica). Da qui la necessità di interventi correttivi, finanziari e strutturali. Sotto il profilo finanziario, la spending review incontra diversi ostacoli oggettivi: l'incomprimibilità della spesa per il personale (non licenziabile, con turn-over bloccato e a stipendi fermi già da anni), la relativa aggredibilità della spesa intermedia (la diminuzione dei prezzi d'acquisto è limitata ad alcune regioni italiane e, qui, è resa difficoltosa dai lunghissimi tempi di pagamento), la contraddizione di dover abbattere la spesa proprio nelle regioni a minore offerta sanitaria, la quale determina quella mobilità, concausa importante dei deficit, che a sua volta garantisce l'equilibrio di quasi tutte le regioni in attivo. Questo è il principale limite anche dell'applicazione dei cosiddetti "costi standard". Sotto il profilo strutturale, le misure proposte sono tutte assai dolorose: revisione dei Livelli Essenziali di 
Assistenza (LEA) (con diminuzione della copertura garantita dei rischi sanitari), universalismo selettivo (con compartecipazione strettamente legata al reddito), aumento della spesa privata, revisione e riduzione della rete ospedaliera (che si scontra con la mission politico-sociale della Sanità), ridiscussione del federalismo sanitario.

\section{Le prospettive europee}

Secondo il report di The Economist Intelligence Unit The future of Healthcare [13], in Europa nel 2023 si spenderà mediamente il $14 \%$ del PIL per la Sanità. Le ragioni dell'aumento dei costi sono ben definite: invecchiamento della popolazione, aumento delle malattie croniche, sviluppo delle tecnologie sanitarie, richieste dei pazienti legate all'aumento delle conoscenze e agli stili di vita, organizzazioni sanitarie focalizzate sui problemi sanitari del passato. È importante cogliere quest'ultimo punto: le strutture sanitarie (ospedali) e il vissuto sociale sono costruiti per vecchi problemi (infezioni, malnutrizione, ecc.) e non per i nuovi (malattie croniche), che richiedono organizzazioni snelle, flessibili e diffuse; i sistemi di finanziamento puntano sui redditi della popolazione attiva e in salute, la cui percentuale si sta abbassando in tutte le società del cosiddetto mondo occidentale.

Le traiettorie che si intersecano sono complicate: la crescita della spesa legata alla politica di aumento della salute delle popolazioni; i modi per rendere trasparenti le priorità sanitarie; la ristrutturazione della Sanità offerta, per mantenere i sistemi universalistici; il ruolo dei medici di medicina generale come "gatekeepers" e coordinatori dei trattamenti; l'importanza degli interventi preventivi e sugli stili di vita; la responsabilità dei pazienti rispetto alla loro salute; la liberalizzazione dei ruoli professionali. I grandi temi all'orizzonte sono: il mix sanitario pubblico/privato; la creazione di un sistema sanitario pan-europeo; una metodologia sanitaria proattiva.

Lo studio presenta cinque scenari "estremi" e contrastanti:

1) la tecnologia trionfa e cura le malattie croniche, la $e$ health ha un ruolo preminente nella gestione dell'organizzazione sanitaria;

2) si crea un sistema sanitario pan-europeo;

3) la prevenzione prende il sopravvento sulla medicina curativa;

4) i sistemi sanitari si focalizzano solo sui membri vulnerabili della società;

5) si afferma una Sanità privata, anche nel finanziamento.

Il primo scenario (Technology triumphant) si basa sulla considerazione che l'organizzazione sanitaria e la salute non sono costi ma investimenti e, quindi, non si teme di spendere il 20\% del PIL per essa. Richiede, però, un alto tenore di produttività stabile e una sostanziale armonia tra pazienti, fornitori e pagatori. Inoltre, è piuttosto improbabile che la tecnologia da sola possa risolvere le malattie croniche: esse sono per definizione mutevoli e altre potrebbero sostituire quelle sconfitte.

Il secondo scenario (Europe united) prevede che la risposta sanitaria sia basata su una rete europea di centri di eccellenza. Richiede armonia tra gli Stati nel finanziamento e nella revisione delle reti ospedaliere. Nell'assistenza territoriale aumenta il ruolo dell'infermiere in diagnosi e prescrizione. Ai pazienti è richiesto di muoversi, anche per migliaia di chilometri, per trovare risposte, peraltro di assoluta qualità, e di essere ben connessi al mondo della $e$-health.

Il terzo scenario (Wellness first) si fonda sulla centralità della prevenzione, che richiede un grande sforzo culturale su medici (e personale sanitario) e pazienti. La responsabilizzazione di entrambi i gruppi viene perseguita anche con misure coercitive e di legge e può dare origine a fenomeni di stigmatizzazione e marginalizzazione sociale. I tempi per un effettivo apprezzamento del miglioramento della salute e del contenimento dei costi sono molto lunghi (decenni) e il programma può essere sovvertito dall'emergere di nuove, sconosciute patologie.

Il quarto scenario (Spotlight on the vulnerable) prende atto dell'insostenibilità dei costi della Sanità e sceglie di proteggere solo i cittadini vulnerabili (anziani $>70$ anni, poveri, malati mentali, minoranze etniche). I costi sono comunque elevati e pagati dai lavoratori in salute e in attività, che dovrebbero anche munirsi di assicurazioni private. Una parte dei medici (e del personale sanitario) e della popolazione potrebbe non accettare quella che loro considererebbero una discriminazione sociale e una degradazione professionale.

Il quinto scenario (Laissez-faire) nasce dall'accettazione degli Stati dell'impossibilità di fronteggiare la sfida e, dunque, di lasciare che il sistema sanitario si autoregoli, andando verso una forma diffusa di privatizzazione. Per evitare rivolte sociali sono necessarie almeno due pre-condizioni: le assicurazioni sono obbligatorie; le assicurazioni non possono rifiutare cittadini su nessuna base discriminatoria. Le prospettive sono quelle di un sistema "managed care" di tipo statunitense. I rischi sono collegati al freno all'innovazione derivante dai prezzi bloccati dei farmaci e device, alle possibili fasce sociali non adeguatamente coperte, all'impreparazione di fronte a possibili pandemie. L'aspettativa di vita potrebbe, allora, cominciare a diminuire.

Non vi è una conclusione definitiva nello studio qui riassunto e gli scenari non sono mutualmente esclusivi. Il sistema sanitario è enorme e frammentato, composto da gruppi che si autodifendono. Tutti concordano sulla difesa dell'universalismo sanitario e sulla necessità di una 
revisione del sistema per garantirne la sostenibilità, ma sono assai divisi sui modi, finanziari e non, per affrontare la sfida. Le difficoltà pianificatorie e programmatiche si scontrano, poi, con le necessità e opportunità politiche e sociali. Non intervenire conduce inevitabilmente verso il quinto o quarto scenario illustrato poc'anzi. Le scelte riguardano il livello di spesa accettabile, l'efficienza del sistema, la coesione sociale sull'efficacia raggiungibile e il ruolo centrale dell'Information and Communication Technology (ICT).

\section{Le prospettive italiane}

Gli scenari futuri delineati per l'Italia si basano sull'analisi delle aree di possibile discontinuità ambientale [14]: la disponibilità complessiva e le fonti della disponibilità finanziaria; i confini del welfare e la mission delle aziende; la cultura, i profili di consumo, la propensione alla mobilità dei pazienti; la trasformazione del capitale umano e dei modelli organizzativi delle aziende; la rivoluzione tecnologica e le nuove architetture infrastrutturali; la strutturazione (ospedali versus territorio) del SSN. Il focus principale è sull'evoluzione delle aziende sanitarie.

La ricerca dell'Università Bocconi [15] abbina a quelle aree di discontinuità altrettanti scenari, indagati con la metodologia NGT (Nominal Group Technique). Questi, a loro volta, sono condensati in quadri di sintesi costituiti da assi ortogonali tridimensionali, i "cubi del futuro": a) "assetti istituzionali e finanziari", in cui si indagano le interdipendenze tra evoluzione dei sistemi di finanziamento, mission aziendali e profili di consumo dell'utenza; b) "ambiti di cura, organizzazione e mobilità dei pazienti", in cui il tema predominante è quello del case manager; c) "infrastrutture, tecnologie, modelli di Sanità", in cui si analizzano le correlazioni tra questi elementi e i modelli di trasformazione del bisogno in domanda.

Il primo cubo connette le diverse spinte in tema finanziario (tassazione, assicurazioni pubbliche o private, outof-pocket) e il loro mix con le caratteristiche di un'utenza sempre più variegata (pazienti fragili, tradizionali, competenti o evoluti) e su cui il tipo di finanziamento ha effetti selettivi (la spesa diretta aumenta l'attenzione dell'utente per qualità e percorsi di cura) e con le mission aziendali rivolte al governo della produzione, a quello dei consumi socio-sanitari o sanitari globali ovvero alla tutela della salute, per trarre tre possibili prospettive di evoluzione delle aziende sanitarie: di nicchia, differenziate (occupano solo alcuni segmenti di mercato), universalistiche. Non viene affrontato il tema del rapporto tra modelli di separazione o di integrazione delle funzioni ospedaliere e territoriali.
Il secondo cubo integra le trasformazioni degli ambiti di cura (rete ospedaliera specialistica, ospedale di prossimità, strutture intermedie, cure primarie), la traiettoria di territorializzazione e la possibile dicotomia tra prevalenza delle strutture intermedie o delle cure primarie con la crescente propensione alla mobilità dei pazienti, seppure nella loro frammentazione (pazienti local, glocal o global), e con gli effetti selettivi sulla tipologia di casemanager (medici, infermieri, care-giver personale, individuo), che varia secondo le patologie, le competenze e le situazioni sociali. Ciò ha effetti determinanti sulla definizione delle aziende come: a) aziende di prossimità, dedicate alle cure territoriali e prive di specialità; b) aziende glocal, costruite attorno alla doppia natura di erogatore locale e competitore nazionale; c) aziende globali, orientate strutturalmente e sistematicamente al mercato della mobilità sanitaria. I punti di difficoltà riguardano l'opportunità del possesso delle specialità, la selezione dei care-givers e la sostenibilità dell'attuale sistema di mobilità interregionale.

Il terzo cubo è costruito dalle interrelazioni tra: a) lo sviluppo della tecnologia (miniaturizzazione, trasportabilità, informatizzazione, connettività), che la rende sempre più "virtuale" e di veloce obsolescenza (7-10 anni), cosicché le grandi tecnologie si concentrano in poche strutture, mentre le altre si distribuiscono nelle strutture anche intermedie e a domicilio (telemedicina); b) le trasformazioni logistiche e infrastrutturali a essa strettamente connesse (da infrastrutture statiche a modulari e virtuali) in una architettura comunque hub \& spokes; c) una posizione della medicina che migra dal modello tradizionale d'attesa (on demand) a quello di diagnosi precoce per specialità (già ora in campo), declinato ulteriormente nella medicina di iniziativa centrata sulla visione globale del medico di medicina generale (come per esempio il chronic care model), ovvero nella medicina "technology driven", collegata alla diffusione di piccole tecnologie individuali e di home testing. Le aziende che si costituiranno a seguito della prevalenza delle interrelazioni descritte potranno assumere i connotati dell'azienda virtuale distribuita a vocazione territoriale e domiciliare, o dell'azienda di diagnosi precoce che favorisce l'empowerment dei pazienti e la selezione della domanda, oppure dell'azienda epidemiologica, costruita sulle basi scientifiche dell'evoluzione delle patologie e delle tecnologie e sui metodi assistenziali a essa necessari (medicina d'iniziativa, mix di tecnologie grandi e diffuse). La pervasività della visione olistica può determinare esclusioni o auto-esclusioni di individui o gruppi discordi rispetto alle priorità aziendali.

Sono tre le principali macro-variabili individuate: a) il ruolo del settore sanitario all'interno del contesto economico-produttivo nazionale: spesa improduttiva o 
motore di sviluppo; b) l'interrelazione tra Sanità, settore sociale e socio-assistenziale: benessere della persona, per determinare i "confini" del sistema sanitario, ma anche il modello di medicina futura (reattiva o proattiva); c) l'approccio alle discontinuità di contesto (struttura della popolazione, contesto tecnologico ed economico), che sono ampiamente "esogene" e difficilmente pronosticabili, ma che hanno un potere di determinazione degli scenari assai rilevante.

Gli scenari hanno probabilità differenziate. Per quanto concerne quelli finanziari, per esempio, gli opinion leader sanitari giudicano più probabile una Sanità pubblica allargata e competitiva sia verso il pubblico sia verso il privato, con un aumento, all'interno del pubblico, delle prestazioni a pagamento (out-of-pocket). Sul fronte della mobilità dei pazienti, potrebbe aumentare il trend attuale di progressione del fenomeno, e a ciò si lega la missione che le aziende sanitarie dovranno studiare per sé in futuro: la presenza di cluster di pazienti sempre più polarizzati e distinti (pazienti evoluti tendenti al self empowerment e pazienti "fragili" caratterizzati da cronicità delle malattie) potrebbe spingere le stesse aziende a differenziarsi. I pazienti evoluti cercheranno il vantaggio economico per le proprie cure, essendo disposti a spostarsi per trovarlo; i pazienti "fragili" faranno invece più riferimento a strutture di prossimità. La partita si gioca, dunque, sulla capacità di attrarre pazienti dall'esterno per strutture ad alta specializzazione, mentre dei secondi le aziende dovranno farsi carico sul territorio, con strutture che si de-specializzino progressivamente. La tecnologia è un driver principale del cambiamento delle abitudini dei pazienti, ma anche dell'organizzazione strutturale, logistica e curativa dei sistemi sanitari.

Stanno cambiando i bisogni legati alla Sanità, le persone, la tecnologia e i modelli organizzativi. L'auspicio è che le politiche da intraprendere si basino sull'analisi del futuro (possibile) e non del passato, mettendo da parte l'abitudine del Paese a ragionare solo sulla contingenza.

\section{Conclusioni}

I sistemi sanitari europei sono a una strettoia comune: da un lato, l'apprezzamento delle nuove potenzialità della medicina è riconosciuto, ma i pazienti chiedono cambiamenti nella fornitura dei servizi; dall'altro, i costi sono in continua espansione sia nei sistemi bismarckiani (fondati su assicurazioni regolate dallo Stato), sia nei sistemi beveridgiani (fondati sulla tassazione e su un servizio pubblico universale).

La spesa sanitaria cresce più del costo della vita, per la struttura demografica delle popolazioni e le loro multipatologie croniche, per il costo di tecnologie e farmaci, per le nuove attitudini dei cittadini. Il contenimento è difficile anche per la crisi economica e sociale intercorrente, che rende più sensibili al taglio delle prestazioni.

Le difficoltà di intervento riguardano, appunto, principalmente la tensione tra efficienza e solidarietà sociale e la sostanza delle organizzazioni sanitarie, enormi e bizantine, fatte di interrelazioni di interessi travestiti e convergenti nel mantenimento dello status quo. L'intensità del sentimento etico e ideologico, unito agli interessi predetti, crea una sorta di "religione" della Sanità che rende difficili gli interventi di revisione.

Tuttavia, lo studio di The Economist Intelligence Unit - Future-proofing Western Europe's healthcare. A study of five countries [16] mostra che in Europa si tentano strade che non sono quelle del razionamento. L'Olanda si è orientata a un finanziamento su base assicurativa, regolata per essere universale: i costi sono stati contenuti, ma i servizi non sono migliorati. La Germania ha puntato sull'integrazione delle cure: vi è stato un miglioramento degli outcomes, con una lieve diminuzione dei costi, ma lo sforzo è molto grande e ha consentito di coprire solo una piccola percentuale della popolazione. Il Regno Unito ha scelto di pubblicare gli esiti delle procedure e delle strutture con effetti positivi sulla qualità delle cure nei casi di studio, ma per diventare generale il metodo richiede una personalizzazione (Patient Recorded Outcomes Measures, PROM). La Danimarca è riuscita dove altri hanno fallito: far funzionare gli Electronic Patient Records (EPR) per tutta la popolazione; gli effetti sono stati una riduzione dei costi e un miglioramento della qualità. La Francia ha rivisto la propria organizzazione, concentrando a livello regionale funzioni altamente coordinate, comprendenti i temi ambientali, la prevenzione, la formazione, le cure mediche e quelle assistenziali LTC.

Queste iniziative mostrano linee d'azione lungo le quali muoversi in modo combinato. Le prospettive rimangono la riorganizzazione strutturale dei sistemi sanitari in termini di concentrazione/dispersione delle tecnologie e interventi sulla base della complessità, il contributo dei pazienti alla prevenzione e agli stili di vita per una crescita culturale e l'intelligente utilizzo della medicina "remota" sulla base di una moderna ITC. Un tema emergente è quello del personale sanitario, chiamato a grandi cambiamenti perché cambiano le tecnologie e le potenzialità, cambiano i pazienti e le loro richieste, cambia la loro vita lavorativa in quanto si allunga (e ciò richiede un nuovo modo di progettare il lavoro per fasce d'età) e in quanto le competenze professionali mutano continuamente, richiedendo una crescita professionale continua e la capacità di travalicare i tradizionali confini delle mansioni originarie.

Conflitto di interessi Nessuno 


\section{Bibliografia}

1. Parker KM, Talbert ML (2003) Laboratory Medicine in the 21th Century. In Ward-Cook KM, Lehmann CA, Scoeff LE, Williams RH Eds. Clinical Diagnostic Technology. The Total Testing Process. Volume 1: The Preanalytical Phase. Washington: AACCPress

2. Cappelletti P (2012) Medicina di Laboratorio e Postmodernità. Riv Ital Med Lab 8:1-15

3. http://www.iom.edu/Reports/2001/Crossing-the-Quality-ChasmA-New-Health-System-for-the-21st-Century.aspx. Institute of Medicine (IOM). Crossing the Quality Chasm: A New Health System for the 21st Century [Accesso 15 giugno 2012]

4. http://www.eesc.europa.eu/self-and-coregulation/documents/codes/private/085-private-act.pdf. Active Citizenship Network, 2002. European Charter of Patients' Rights [Accesso 15 giugno 2012]

5. Dorizzi RM (2009) The Healthcare Imperative: lowering costs and improving outcomes: ancora una volta l'Institute of Medicine traccia la via. RIMeL/IJLaM 5:173-7

6. Lega F (2010) Gli scenari come strumento di pianificazione strategica. In Longo F, Del Vecchio M, Lega F. La sanità futura. Come cambieranno gli utenti, le istituzioni, i servizi e le tecnologie. Milano: EGEA, pp 7-26

7. http://www.camera.it $/ 465$ ?area $=30 \&$ tema $=34 \& I 1+$ controllo+ della+spesa+sanitaria. Parlamento italiano. Camera dei Deputati. Controllo della spesa sanitaria [Accesso 15 giugno 2012]

8. http://www.oecd.org/dataoecd/45/52/43216313.pdf. OECD Health Data 2011. How Does Italy Compare [Accesso 15 giugno 2012]

9. Longo F, Tasselli S (2010) Gli scenari istituzionali e la mission delle aziende. In Longo F, Del Vecchio M, Lega F. La sanità futura. Come cambieranno gli utenti, le istituzioni, i servizi e le tecnologie. Milano: EGEA, pp 113-144

10. http://www.ambrosetti.eu/it/download/ricerche-e-presentazioni/2011/meridiano-sanita-rapporto-finale-2011. The European House Ambrosetti. "Meridiano Sanità. Le coordinate della salute" Rapporto Finale 2011 [Accesso 15 giugno 2012]

11. http://www.cermlab.it/_documents/_argomenti/PROJECTIONS.pdf. CeRM. Le proiezioni della spesa sanitaria SSN. SaniMod 2011-2013 [Accesso 15 giugno 2012]

12. http://www.rgs.mef.gov.it/_Documenti/VERSIONE-I/Attivit-i/Spesa-soci/Attivit--d/2011/Le-tendenze-di-medio-lungo-periodo-del-sistema-pensionistico-e-socio-sanitario-Rapporto_n.12.pdf. Ministero dell'Economia e delle Finanze. Le tendenze di medio-lungo periodo del sistema pensionistico e sociosanitario. Rapporto n. 12 [Accesso 15 giugno 2012]

13. http://www.janssen-emea.com/sites/default/files/The $\% 20$ Future $\% 20$ Of $\% 20$ Healthcare\%20in\%20Europe.pdf [Accesso 15 giugno 2012]

14. Longo $F$ (2010) Le aree di possibile discontinuità ambientale. In Longo F, Del Vecchio M, Lega F. La sanità futura. Come cambieranno gli utenti, le istituzioni, i servizi e le tecnologie. Milano: EGEA, pp 27-37

15. Longo F, Del Vecchio M, Lega F (2010) La sanità futura. Come cambieranno gli utenti, le istituzioni, i servizi e le tecnologie. Milano: EGEA

16. http://www.eucomed.org/uploads/Modules/Publications/ 111005_eiueucomedfutureproofing_healthcarefinalv2web_5101 1.pdf. The Economist Intelligence Unit. Future-proofing Western Europe's healthcare. A study of five countries [Accesso 15 giugno 2012] 